\title{
Effect of Enzyme Preparations with Endo-Xylanase and Exo-Peptidase Activities on the Bread Quality, Dough Properties and Fractional Composition of Wheat Gluten Proteins
}

\author{
A.A. Nevsky \\ Department of biochemical research \\ Federal State Autonomous Scientific Institution 'Scientific \\ Research Institute of the Bakery Industry', \\ Moscow, Russia \\ obishleb@ya.ru \\ G.F. Dremucheva \\ Department of biochemical research \\ Federal State Autonomous Scientific Institution 'Scientific \\ Research Institute of the Bakery Industry', \\ Moscow, Russia

\section{I.A. Velikoretskaya} \\ All-Russian Scientific Research Institute for Food \\ Biotechnology - a branch of the Federal State Budgetary \\ Science Institution of the Federal Research Center for \\ Nutrition, Biotechnology and Food Safety, \\ Moscow, Russia
}

Abstract - Enzymes (EN) are widely used in many countries with purpose to adjust the quality of flour in baking and milling industries. It is known that the combined application of EN with endo-xylanase and exo-peptidase activities provides a higher positive effect in the bread quality compared to the use of EN with pure endo-xylanase activity. However, the factors responsible for this effect are not found. In this regard, the aim of the study was to determine the role of complex EN with endo-xylanase and exopeptidase activities in improving the quality of bread from wheat baking flour. We compared the effect of EN with endo-xylanase (EN1) and complex EN with endo-xylanase and exo-peptidase activities (EN2) on the properties and fractional composition of gluten proteins from wheat flour and dough. It was established that EN1 and EN2 improved physicochemical and organoleptic parameters of bread quality, formed a more elastic and lighter crumb with uniform fine and thin-walled structure, and also influenced on the aggregation state of protein molecules of flour the amount and properties of gluten, the transformation of protein fractions in the dough making process. EN1 and EN2 increased the degree of hydration and aggregation of gluten proteins, the strength of their combination during dough mixing, which resulted in the increased amount of wet and dry gluten, the total amount of the fraction of spirit-soluble and alkaline-soluble proteins, and the hydration ability of gluten. It was established

\author{
N.V. Tsurikova \\ All-Russian Scientific Research Institute for Food \\ Biotechnology - a branch of the Federal State Budgetary \\ Federal State Autonomous Scientific Institution 'Scientific \\ Research Institute of the Bakery Industry', \\ Moscow, Russia \\ M.V. Nosova \\ Federal State Autonomous Scientific Institution 'Scientific \\ Research Institute of the Bakery Industry', \\ Moscow, Russia \\ D.M. Borodulin \\ Doctor of Technical Sciences, Head of the Department \\ technological design of food production \\ Kemerovo State University \\ Kemerovo, Russia Federation \\ borodulin_dmitri@list.ru
}

that endo-xylanase of $\mathrm{EN}$ indirectly reduces the degree of peptization of gluten proteins during the dough fermentation and proofing, as evidenced by less degree of dry gluten reduction during these stages of dough preparation compared to the control without EN. The difference in the effect of EN1 and EN2 was demonstrated during the dough fermentation and proofing. The obtained data on the role of $\mathrm{EN}$ with endo-xylanase and exopeptidase activities in the modification of gluten and the fractional composition of proteins will allow to design the EN compositions with expected technological effect to improve the efficiency of flour and bread quality control.

Keywords-gluten, wheat flour, fractional protein composition, enzyme preparation, endo-xylanase activity, exopeptidase activity.

\section{INTRODUCTION}

At present, wheat, supplied to flour mills, does not always have satisfactory baking properties. As a result, the quality of the flour produced from wheat has to be adjusted either at the production of flour or in the industrial bakeries, in particular by the application of improvers. Increased requirements for food safety led to a decrease of the number of chemical 
additives used, the widely accepted alternative being enzymes (EN). EN are widely used, including those with endo-xylanase and proteolytic activities in many countries to improve the baking perfromance of flour. The conducted studies showed the effectiveness of the use of complex EN with endo-xylanase and exo-peptidase activities in the dough making processes for bakery products from wheat flour.

In the USSR, the baking industry used ENs, produced domestically, however due to the liquidation of Russian plants, producing FP, the enzymes, produced by foreign companies are present on the market nowadays. On the basis of new highly active producers obtained at All-Russian Scientific Research Institute for Food Biotechnology, a pilot batch of the EN Protozim with combined activities was produced. The studies of technological properties Protozim in the production of bakery products are made in the Department of biochemical research of Federal State Autonomous Scientific Institution 'Scientific Research Institute of the Bakery Industry'.

For the practical implementation of Protozim in the baking industry, it is necessary to determine the influence of EN not only on the quality of bread, but also on the properties of gluten, including the comparable study with the foreign alternative, which is widely used in the production of bread.

Physical and physicochemical properties of gluten play the most important role in the formation of the quality of wheat bread, because they influence on the composition and structure of its main components - proteins, as well as other factors, including EN application with xylanase, protease nd other activities. In this connection, it is advisable to establish the joint role of Protozim endo-xylanase and exo-protease in the transformation of flour proteins in the process of bread preparation.

The main part of wheat flour proteins $(80 \%)$ is represented by gluten proteins - gliadins (40-50\%) and glutenins (34-42\%), the amount of other proteins represent with albumins as 5.7$11.5 \%$, and globulins as 5.7-10.8\%.

The importance of glutenins and gliadins in the formation of gluten has been established intensively, the largest being of high-molecular glutenins, whose number is only $8-10 \%$ of the total protein mass. It is well known that high-molecular glutenins provide the strength and elasticity, and low-molecular glutenins and gliadins are responsible for viscous properties of gluten.

Water-soluble proteins do not have water-absorbing capacity. Thus, the removal of water-soluble protein from wheat flour dramatically increases the hydration of flour, and the dough formation proceeds more slowly. It has been shown that aqueous extracts of wheat flour, in addition to albumins, usually contain a certain amount of gliadin, and, possibly, some globulins. When the dough is mixed, the proteins of the flour are hydrated, resulting in a significant increase in their volume. As a consequence of adhesion, sticking of hydrated proteins and the formation of a continuous protein network of the dough glutens occur. A good correlation between the number of gliadins aggregated by the disulfide bonds and the dough strength was found It is noted that the ability of disulfide bonds to regroup during the dough mixing is an important factor in the development of gluten viscous-elastic properties.

The process of the dough kneading together with the hydration of proteins causes a profound effect in the molecules of the latter. For example, during the dough kneading according to Bushuk W. (1973), the amount of proteins soluble in $0.05 \mathrm{M}$ acetic acid with the molecular weight of more than 150,000 sharply increases, which corresponds to low molecular weight glutenin. Further studies Bushuk W. (1973), confirmed that the increase in the solubility of proteins in acetic acid is precisely due to the glutenin fraction. As the dough kneading continues, the amount of soluble glutenin increases, and the insoluble one decreases accordingly.

The change in protein substances during the dough fermentation is influenced by the products of alcoholic fermentation, acidity increase, as a result of which is the swelling and solubility of gluten proteins, primarily gliadin, increase. The proteases play a certain role, causing depolymerization of protein macromolecules due to the hydrolysis of peptide bonds. At the same time, the yield of gluten washed out from the dough is lowered, but it is stronger than the gluten washed out from the dough after kneading. Glutathione of baking yeast restores disulfide bonds, which relaxes gluten.

The results of numerous studies indicated an extreme instability in the aggregation equilibrium of protein macromolecules, which is disturbed by $\mathrm{pH}$, the presence of various salts having buffer properties in the medium, and other factors. There is no precise molecular mechanism for the formation of viscoelastic properties of dough from wheat flour.

The gluten protein has a high ability to absorb non-protein compounds contained in the flour, and introduced during the dough kneading, which changes the gluten structural and mechanical properties. For example, the role of non-starch polysaccharides (pentosans, $\beta$-glucans) has been established that have a significant water-binding capacity in additional gluten hydration during dough kneading. According to Bushuk W. (1973), after the dough kneading, $26.4 \%$ of water absorbs undamaged and 19.1\% - damaged starch grains, 32.1\% being found in gluten and $23.4 \%$ being associated with pentosans. Insoluble arabinoxylans (Insol.-ARX) - highly branched polymers prevent the formation of a continuous glutinous matrix, as a result of which its uniformity is impaired and its elasticity is reduced. During the dough fermentation, the envelopes of gas bubbles are destroyed, causing their coalescence. As a result, the bread volume decreases and the crumb properties deteriorate.

Hydrolysis of Insol.-ARX increases the amount of mediumand high-molecular-weight water-soluble arabinoxylans (Wsol.-ARX), solubility of which in the aqueous medium increases after the short-term dough kneading and further increases as the rest time grows. So, according to Bushuk W. (1973) with $0.66 \%$ Wsol.-ARX content in wheat flour, it is $0.98 \%$ after kneading, and $1.32 \%$ after 3 hours of dough rest. At the same time, the water absorbed by Insol.-ARX is released and its redistribution occurs between the dough structural components, mainly between the gluten proteins and pentosans. Additional hydration of gluten proteins increases its 
extensibility, improves the dough elastic properties. It is suggested that Wsol.-ARX form a spatial structure with flour gluten, and is also part of the monomeric film of gas bubbles that strengthens and stabilizes it, preventing coalescence.

The role of proteolytic enzymes in bread technology is well known. With the introduction of low doses of proteolytic EN, the amount of gluten washed out from the dough is increased due to the growth of its hydration, which leads to a more complete formation of gluten and, consequently, to an increase in its yield.

Exo-peptidases from wheat flour are important in the technology of bakery products, which are characterized by a sequential "terminal" rupture of peptide bonds, accompanied by the accumulation of low-molecular nitrogenous substances with a carbonyl or amine group. With exo-peptidases, the decrease in the viscosity of hydrated gluten and the increase in the content of amino acids in the dough are attributed. Amino acids are essential for the life of yeast and the reaction of melanoidin formation. The use of exo-peptidases improves the taste and smell of bread, the color of crusts of baked goods

It is known that the combined use of EN with endo-xylanase and proteolytic activities provides a higher quality improving effect of bread compared to their separate application.

The aim of the research was to study the effect of complex EN possessing endo-xylanase and exo-peptidase activities on the quality of bread from wheat baking flour of the highest grade, as well as defining the EN determinant with endoxylanase and exo-peptidase activities in comparison with EN only with endo- xylanase activity in improving the quality of bread and transforming the protein in the dough preparation.

Work in this direction is relevant, since the unstable quality of the grain requires adjusting the quality of flour in flour milling and baking plants, which is most advantageous through the use of EN

To achieve this goal, we studied the effect of EN on the quality of bread, the properties of gluten and the fractional composition of wheat flour and dough proteins.

\section{MATERIALS AND METHODS}

The study subjects were wheat flour of the highest grade, enzyme preparation Pentopan Mono (EN1, NOVOZYMES, Denmark), enzyme preparation Protozim (EN2, All-Russian Research Institute of Food Biotechnology, experimental lot, $\mathrm{RF})$.

\section{A. Laboratory base}

Laboratory research was carried out on the basis of the Center of Technologies, Biochemical and Microbiological Research (Federal State Association of Scientific Organization, Research Institute of Chemical Industry); the study of the fractional composition of wheat gluten proteins was carried out in the laboratories of the All-Russian Scientific Research Institute of Food Biotechnology.

\section{Raw materials used in the research}

The following raw materials were used in the research:
- wheat flour of the highest quality (State Standard Of Russia 52189-2003);

- pressed bakery yeast (State Standard Of Russia 547312011);

- 'Extra' grade table salt (State Standard Of Russia 51574 2000);

- enzyme preparation Pentopan Mono (EN1, NOVOZYMES, Denmark);

- complex enzyme preparation Protozim (EN2, All-Russian Research Institute of Food Biotechnology, experimental lot, $\mathrm{RF}$;

- drinking water (Sanitary (sanitary-epidemiological) rules and regulations 2.1.4.1074-01).

Indicators of flour quality. Wheat bakery flour of the highest grade was characterized by the following indicators: mass fraction of moisture $-14.5 \%$; gluten content $-28 \%$; quality of gluten -50 conventional units by the IDK device; characteristic gluten - II quality group, satisfactorily strong; the falling number is $290 \mathrm{~s}$; acidity -2.2 degrees.

EN characterization: EN1 - endo-xylanase activity - 2500 FXU (W)/g; EN2 - endo-xylanase activity - 1075 units $\mathrm{g}^{-1}$; XA $\mathrm{g}^{-1}$, exo-peptidase -350 units $\mathrm{g}^{-1}$.

The quality indicators of other raw materials used in the studies met the requirements specified in the technical documentation.

\section{B. Methods to prepare dough and bread}

The dough was prepared in a single-phase method according to the recipe, \%: wheat flour of the highest grade -100.0 , pressed bakery yeast -3.0 , table salt 1.5 , drinking water 55.75. The dough was kneaded for $5 \mathrm{~min}$., the dough fermentation time was $60 \mathrm{~min}$. at the temperature of $30 \pm 2^{\circ} \mathrm{C}$. After that the dough pieces were molded into balls and placed into the proofer. The proofing was carried out at the temperature of $36-38^{\circ} \mathrm{C}$ and relative humidity of air was $76-78 \%$ for $53-55$ min. The baking was carried out at the temperature of 210 $220^{\circ} \mathrm{C}$ for 25 minutes. Experimental probes were added with $\mathrm{EN} 1$ and EN2 in an amount of 5 XA units for $1 \mathrm{~g}$ of flour.

When examining the effect of EN1 and EN2 on the amount and properties of gluten, the dough was kneaded from $200 \mathrm{~g}$ of flour, the gluten was washed from dough samples selected in the amount of $40 \mathrm{~g}$ immediately after kneading and after 120 minutes of fermentation.

When determining the fractional protein composition, the dough was prepared from $300 \mathrm{~g}$ of flour, after kneading it was fermented for 60 minutes at the temperature of $30 \pm 2^{\circ} \mathrm{C}$, after the dough pieces were molded into balls and placed into the proofer. Conditions of proofing: temperature $-36-38^{\circ} \mathrm{C}$, relative humidity of air $-76-78 \%$, duration $-53-55 \mathrm{~min}$. The dough probes were taken in the amount of $40 \mathrm{~g}$ immediately after kneading, then after the dough fermentation and after the dough pieces proofing. 


\section{The research methods used in the work}

In studying the properties of raw materials, semi-finished products and the quality of bakery products, the conventional physicochemical and organoleptic methods were used, as well as the special methods introduced in the text of the work.

\section{Methods for investigating the properties of raw materials}

All probes of high-grade wheat baking flour were analyzed for organoleptic and physicochemical parameters: moisture, acidity, number of drops, quantity and quality of crude gluten.

The moisture content of the flour was determined according to GOST 9404-88, the acidity of flour - according to GOST 27493-87, the number of drops - according to GOST 2767688 , the content of crude gluten - on the device MOK-3M in accordance with GOST 27839-2013, the properties of crude gluten - on the device IDK-3M according to GOST 278392013.

The pressed yeast was analyzed by the time of the dough rise in accordance with GOST R 54731-2011.

Table salt, enzyme preparations and drinking water were evaluated organoleptically in accordance with the current documentation.

\section{E. Methods for assessing the quality of bread}

Bread was analyzed after 14-16 h after baking by organoleptic and physicochemical parameters: crumb humidity, crumb acidity, crumb porosity, specific volume and form stability.

The specific volume of bread was determined in accordance with GOST 27669-88 and expressed in $\mathrm{cm}^{3} \mathrm{~g}^{-1}$, the moisture content of the crumb was determined according to GOST 21094-75, the acidity of the crumb - by the accelerated method according to GOST 5670-96, porosity of the crumb - according to GOST 5669-96, form stability - according to GOST 2766988. Organoleptic indicators of bread quality were assessed in accordance with GOST 5667-65 and GOST 27669-88.

\section{F. Methods for investigation of the properties of semi-finished products}

The quantity and quality of crude dough gluten were determined in accordance with the procedure given in GOST 27839-88, the amount of dry gluten, according to the procedure given in GOST R 54478-2011; the hydration ability of gluten was determined by the method described in the manual.

\section{Special research method for determination of the fractional composition of gluten proteins}

The fractional composition of flour and dough proteins was determined by the probe successive dissolution at $0.05 \mathrm{n} . \mathrm{NaCl}$, $70 \%$ ethanol and $0.05 \mathrm{n}$. NaOH. A sample probe weighing 2-4 $\mathrm{g}$ was suspended in $20 \mathrm{~cm}^{3} 0.05 \mathrm{n}$. NaCl for an hour on a shaker, and then left overnight in the refrigerator. The dispersion was then centrifuged for $15 \mathrm{~min}$. at $6,000 \mathrm{rpm}$ and the supernatant was poured into a $100 \mathrm{~cm}^{3}$ volumetric flask. The precipitate was again poured with the same amount of solvent, washed, centrifuged and poured into the same volumetric flask. The solution was brought to the mark, and the precipitate was poured with another solvent and all operations were repeated. Protein in the centrifuges was determined by the Lowry (Lowry, 1951), the results were expressed as the percentage of the total content of alkali-soluble proteins in the solution.

\section{RESULTS AND THEIR DISCUSSION}

An important indicator of the baking advantage of wheat flour is its ability to form elastic dough. A lot of experimental data on the role of structural components of flour in the formation of the dough rheological properties has been accumulated, incl. the importance of the degree of hydration of proteins and the role of non-starch polysaccharides.

When studying the effect of enzyme preparations with endoxylanase (EN1) and endo-xylanase and exo-peptidase activities (EN2) on the quality of bread, the quantity and quality of gluten washed from dough after the dough kneading and fermenting, as well as the fractional composition of wheat flour and dough proteins after kneading, fermentation and proofing of the dough pieces. A flour probe with satisfactorily strong gluten was used, (see test) not providing the formation of an elastic crumb with uniform and fine porosity. In practice, EN with endo-xylanase activity is used to improve these parameters.

The results of the influence of EN1 and EN2, used in the optimal dosage (5.0 units ha g-1 flour) on the quality of bread made from wheat flour of the highest grade with a satisfactorily strong gluten showed that EN1 and EN2 provided an improvement in physical, chemical and physicochemical and organoleptic parameters of bread quality: specific bread volume increased by 12.6 and $11.7 \%$, porosity of crumb by 5.1 and $5.1 \%$, form stability of bread - by 10.0 and $6.7 \%$ in comparison with the control one. Enzyme preparations contributed to the formation of a more elastic, lighter with uniform thin-walled porosity crumb compared to the control one. The somewhat less form stability of the sample with EN2 in comparison with the form stability of the grain with EN2 is probably due to the action of the exo-peptidase EN2.

Gluten of wheat dough is a multi-component structure that has a spatial shape and characteristic properties that affect the volume, form, structure of the crumb porosity - uniformity, size and thickness of the pores, and elasticity of the crumb.

The study of the characteristics of wheat dough gluten when using enzyme preparations that improve the quality of bakery products is suitable for adjusting the dough preparation and modeling of new technological systems.

The basic stages of the dough preparation, during which the properties of the protein-proteinase complex of flour change and the dough properties, and, accordingly, the quality of the bread are formed, are the dough kneading and fermenting.

In this connection, at the next stage of the study, the effect of enzyme preparations with endo-xylanase and exo-peptidase activities on the quantity and quality of gluten, the fractional composition of wheat flour proteins was determined.

Enzyme preparations were used in the optimal amount, providing the maximum effect of improving the organoleptic 
and physicochemical parameters of the bread quality: EN1 in the amount of 5.0 units XA g ${ }^{-1}$ of flour and complex EN - EN2 with endo-xylanase activity in an amount of 5.0 units $\mathrm{XA} \mathrm{g}^{-1}$ of flour and exo-peptidase activity in an amount of 24.0 units g-1 of flour.

The process of gluten formation begins after the contact of flour with water and hydration of proteins. When washing, gluten, starch, water-soluble proteins, sugars, etc., are removed together with water, and hydrated gluten proteins stick together into larger particles held by different bonds. More firmly connected protein macromolecules in the gluten structure increase the mechanical strength of gluten

EN with endo-xylanase activity addition leads to the hydrolysis of insoluble arabinoxylans to form water-soluble arabinoxylans. According to the scientific and technical literature, water absorbed by insoluble arabinoxylans is thus released and redistributed between the structural components of the dough, mainly between the gluten proteins and pentosans. So, endo-xylanases promote the creation of a continuous structure and strengthening of gluten.

The conducted studies showed that EN1 and EN2 during the dough kneading influence the process of gluten dough formation from wheat flour of the highest grade. The degree of influence of EN was approximately the same: after the dough kneading, the amount of crude dough gluten with EN1 and EN2, compared to the control one was more - by 2.5 and $2.2 \%$, that of dry gluten - by 4.6 and $3.4 \%$. The gluten hydration capacity of the experimental probes with EN1 and EN2 was higher than that of the controls at 6.0 and $5.0 \%$, respectively.

The higher hydration capacity of the gluten washed from the test dough probes testifies to a higher degree of water absorption by the gluten proteins during dough kneading, a larger amount of dry gluten - about the formation of a more resistant to mechanical action and, therefore, a stronger gluten. It is obvious that the increase in the amount of washed crude gluten, as well as the increase in the hydration ability, and the amount of dry gluten are the result due to the action of EN1 and EN2.

After 120 minutes of fermentation, the amount of crude gluten washed from the control probe decreased by $16.2 \%$; from probes with EN1 and EN2 - to a lesser extent - by 15.4 and $14.5 \%$, the amount of dry gluten of the control probe decreased - by $17.2 \%$, probes with EN1 - by $12.1 \%$, with EN2 - by $14,4 \%$. Consequently, it can be assumed that PF1 and PF2 indirectly reduce the degree of peptization of gluten proteins during fermentation and proofing of dough pieces (DP).

The hydration ability of gluten of the control probe after the dough fermentation increased by $10.1 \%$, the probes with EN1 and EN2 decreased by 6.8 and $5.1 \%$, respectively. The hydration ability of gluten, washed from a control dough probe after 120 minutes of fermentation, was $180 \%$, i.e. it approached the value of the hydration ability of gluten of experimental probes, washed immediately after the dough kneading (177 and $175 \%$ ). Consequently, the degree of hydration of the gluten proteins of the test probes, achieved after the dough kneading with EN1 and EN2, is achieved in the control probe only after 120 minutes of fermentation.

The data obtained are consistent with the results of Bushuk W's studies (Bushuk W., 1973) on increasing the hydration of proteins during the dough fermentation as a result of hydrolysis of Insol.-ARX and an increase in the amount of Wsol.-ARX.

It is known that the change in protein substances during the dough fermentation is affected by products of alcoholic fermentation, acidity growth, which increases the swelling and solubility of gluten proteins. The decrease in the amount of gluten during the dough fermentation is due to the peptization of gluten proteins under the influence of proteolytic enzymes of flour, organic acids formed as a result of the vital activity of the microflora of the dough, and other factors. The degree of changes occurring during the dough fermentation depends also on the duration of the process.

A smaller degree of reduction in the amount of crude and dry gluten in the test probes compared to their content in the control probe indicates that the gluten proteins of the experimental probes are less prone to destruction than those of the control probe. Consequently, even after the dough fermentation, the gluten of the experimental dough probes with EN1 and EN2 was stronger than that of the control probe. A slightly smaller amount of dry gluten after the probe with EN2 fermentation in comparison with the EN1probe is obviously a consequence of the action of exo-peptidase EN2, which causes a moderate proteolysis of flour proteins.

Thus, the different directionality and the degree of change in these indicators of the control and test samples testify that indirectly, EN1 and EN2 reduce the degree of peptization of gluten proteins during the dough fermentation.

The obtained data suggest that after kneading more free water remains in the dough test probe than that of in the experimental probes. Since the gluten is a three-dimensional structure, the volume of which increases as the dough fermentation is increased by the action of carbon dioxide produced as a result of the vital activity of the baker's yeast, then obviously the availability of the protein components of the glutinous net for the contact with free water rises, i.e. free water can be bound with non-hydrated proteins when dough is kneaded.

Probably, when kneading the experimental dough probes as a result of the action of endo-xylanases EN1 and EN2, the maximum degree of hydration of gluten proteins is achieved, and a decrease in the hydration ability of the dough during fermentation is a consequence of a decrease in the amount of gluten proteins due to their peptization.

At the next stage of the study, the amount of protein fractions was determined: water-soluble (WSP+ SSP), spiritsoluble (SpSP) and alkali-soluble (ASP) in the dough after kneading and fermentation, then DPs proofing.

The results showed that the addition of EN1 and EN2 influenced the amount of protein fractions during the dough preparation. So, after the control dough kneading, the amount of water-soluble proteins (WSP+SSP) decreased with the probes with EN1 and EN2, as compared to flour by 1.2; 24.4 
and 24.0\%; spirit - soluble (SpSP) - by 45.8; 44.4 and $44.1 \%$, and alkali-soluble (ASP), on the contrary, increased by 56.2 ; 71.5 and $70.9 \%$ respectively.

The total amount of gliadin and glutenin gluten proteins (SpSP + ASP) after kneading the experimental probes with EN1 and EN2 was larger $(81.1$ and $81.0 \%)$ than that in the control probe $(71.5 \%)$.

After the dough kneading, the ratio of the amount of watersoluble protein fraction to alkali-soluble one (WSP+SSP)/ASP and the ratio of spirit-soluble proteins to alkali-soluble proteins (SpSP/ASP) changed in comparison with the flour.

The ratio (WSP+SSP)/ASP compared with the flour (0.73) in the control probe after kneading decreased to 0.46 , in the probe with EN1 - to 0.32 in the probe with EN2 - to 0.33 , the ratio of SpSP/ASP (in the flour - 1.2) in the control probe was reduced to 0.42 , in the probe with EN1 - up to 0.39 in the probe with EN2 - up to 0.39), respectively. A smaller ratio (WSP+SSP)/ASP and SpSP/ASP in the test probes compared with the control one shows that the degree of aggregation of gluten proteins during the kneading of the dough experimental probes was higher than that of in the control.

The obtained data indicate that during the dough kneading, the aggregation of a part of the WSP, SSP with the formation of the ASP occurred, and in the experimental probes to a greater extent, which is probably due to the action of endo-xylanases of the EN, which promotes the formation of a stronger gluten with a continuous structure.

After the dough fermentation and DPs proofing of the control probe, the amount of water-soluble proteins, as compared with the amount of them after kneading, decreased by 13.8 and $9.7 \%$, respectively, in the probe with EN2 - by 13.7 and $1.0 \%$, but in the probe with EN2 after fermentation it increased by $4.2 \%$, and after DPs proofing decreased by $10.0 \%$.

It is known that the amount of water-soluble proteins during the dough fermentation depends on many factors: the degree of proteolysis of proteins due to the activity of the protease of flour and introduced EN, the acidity of the medium and the amount of low molecular substances formed and consumed by yeast and lactic acid bacteria, etc. In addition, both partial agglomeration and reassociation of gluten proteins can occur.

The ratios (WSP+SSP)/ASP and SpSP/ASP after the dough fermentation and DPs proofing, as compared with the ratio after the dough kneading, varied in the probes (Table 4), which agrees with the known data that the fermenting dough is a system the functioning and structure of which is influenced by many factors, i.e. degree of hydration and peptization, ability of proteins to agglomeration and reassociation, etc.

At the same time, the total content of gluten proteins (SpSP+ASP) (Table 3) was higher in all stages of the dough preparation in experimental probes with EN1 (81.1-83.7\%), with EN2 $(80.2-82.9 \%)$ than in the control probe $(75.3-$ $77.7 \%)$.

Comparison of the results of studying the changes in the fractional composition of proteins during the preparation of the wheat dough and the properties of the gluten washed from the dough after kneading and fermentation are consistent with each other and suggest that the endo-xylanase of the ENs not only contributes to the formation of a more stable and continuous gluten structure in the dough kneading, but also indirectly reduces the degree of peptization of gluten proteins during the dough fermentation and the DPs proofing, which is confirmed by a smaller ratio of $\mathrm{SpSP} / \mathrm{ASP}$ in the probe with EN2 in comparison with EN1.

Different influence of EN1 and EN2 on the amount of dry gluten after the dough fermentation, and also somewhat lower content of gluten proteins after DPs proofing, but almost equal to the hydration capacity of the dough probes, are clearly the result of the action of exo-peptidase EN2: it causes mild proteolysis of proteins and would increase the amount of lowmolecular nitrogenous substances assimilated by baker's yeast. It can be assumed that the effect of exo-peptidases EN2 increases the fraction content of highly hydrated gluten protein, which does not pass into the aqueous solution and is not released during gluten washing, but which in this more mobile state, is more evenly distributed both throughout the mass of the gluten and the dough.

The results of the study showed that the use of EN1 and EN2 helps to accelerate the formation of dough gluten made from wheat flour with satisfactorily strong gluten. As a result, it is expedient to shorten the duration of the dough fermentation when used.

\section{CONCLUSIONS}

1. It has been established that EN1 with endo-xylanase and EN2 with endo-xylanase and exo-protease increase the degree of hydration of gluten proteins and contribute to the hardening of its structure.

2. It has been established that EN with endo-xylanase activity indirectly reduces the degree of peptization of gluten proteins during the dough fermentation, which confirms the higher amount of dry gluten in the experimental probes compared to the control one.

3. The degree of action of EN2 with endo-xylanase and exo-protease activities on the properties of gluten and the fractional composition of proteins differs from the effect of PF1 with endo-xylanase activity.

4. It is assumed that the exo-peptidase of EN2 increases the content of highly hydrated gluten protein, which is not released when washing gluten. The protein in this state is more mobile, more evenly distributed throughout the mass of gluten and dough.

5. Scientific novelty of the work. The effect of EN1 with endo-xylanase and EN2 with endo-xylanase and exoprotease activities on hydration, the amount of crude and dry gluten during the dough kneading from wheat flour with satisfactorily strong gluten, and also the conversion of protein fractions of gluten during the dough fermentation and DPs proofing.

6. The practical significance of the work carried out is that the data obtained on the role of EN with endo-xylanase 
and exo-protease activities in the modification of gluten and the fractional composition of proteins will allow the creation of EN compositions with the expected technological effect through which the efficiency of flour and bread quality management would be increased.

The work was carried out within the framework of the Russian Federal Association of Scientific Organizations, Research Work № 0593-2014-0016 'To develop a methodology for creating import-substituting technologies for complex improvers based on biotechnological characteristics of domestic enzyme preparations for improving the quality of bakery products from flour with various baking properties'.

\section{LIST OF ABBREVIATIONS}

- EN - enzyme preparation;

- $\mathrm{WAC}$ - water absorption capacity;

- InSol-ARX - insoluble arabinoxylans;

- Sol - ARX -soluble arabinoxylans;

- EN1 - enzyme preparation Pentopan Mono,

- NOVOZYMES, Denmark;

- EN2 - enzyme preparation Protozim, Russia;

- DP - dough piece;

- WSP - water soluble protein;

- $\mathrm{SSP}$ - salt soluble protein;

- $\mathrm{SpSP}$ - spirit soluble protein;

- $\quad$ ASP - alkali soluble protein

\section{References}

[1] M.M. Bueno, R.C.S. Thys and R.C. Rodrigues, "Microbial enzymes as substitutes of chemical additives in baking wheat flour-Part I: individual effects of nine enzymes on flour dough rheology", Food Bioprocess Technol, vol. 9(12), 2016, pp. 2012-2023.

[2] S. P. Cauvain ed. by, "Breadmaking. Improving quality", 2nd ed, Woodhead Publishing Limited, 2012, 802 p.

[3] P.A. Caballero, M. Gomez and C.M. Rosell, "Improvement of dough rheology, bread quality and bread shelf-life by enzymes combination", Journal of Food Engineering, vol. 81(1), 2007, pp. 45-50.

[4] N. Chaudhary, P. Dangi and B.S. Khatkar, "Relationship of molecular weight distribution profile of unreduced gluten protein extracts with quality characteristics of bread", Food Chemistry, vol. 210(1), 2016, pp. 325-331.

[5] K.N. Chizhova, "The gluten protein and its transformation in the process of baking", Moskva, Food industry, 1979, p. 135.

[6] K.N. Chizhova, T.P. Shkvarkina, and others, "Technochemical control of bakery production", Moskva, Food Industry, 1975, p. 479.

[7] G.F. Dremucheva, O.E. Karchevskaya, N.A. Kindra and S.A. Smirnova "Bakery properties of wheat and rye flour from the grain of the crop of 2014-2015", Bakery of Russia,vol. 4, 2016, pp. 16-19.

[8] G.F. Dremucheva, A.A. Nevsky, N.G. Bessonova, I.A. Velikoretskaya, N.V. Tsurikova and A.P. Sinitsyn,. "Influence of the domestic enzyme preparation with endo-xylanase activity on the baking properties of wheat flour and the quality of bread”, Khlebopechenie Rossii, vol. 4, 2017, pp. 35-38.

[9] C. Ferrero, "Hydrocolloids in wheat breadmaking: A concise review", Food Hydrocolloids, vol. 68, 2017, pp.15-22.

[10] "Food ingredients in the production of bakery and flour confectionery", Monograph, Moskva, DeLi-plus, 2013, 527 p.

[11] M. G. Ganzle, J. Loponen and M. Gobbetti, "Proteolysis in sourdough fermentations: mechanisms and potential for improved bread quality", Trends in Food Science \& Technology, vol. 19(10), 2008, pp. 513-521.

[12] H. Goesaert et. al, "Wheat flour constituents: How they impact bread quality, and how to impact their functionality", Trends in Food Science and Technology, vol. 16(1-3), 2005, 12-30 pp.

[13] J. D. Figueroa-Cárdenas, A. Escalante-Aburto, J. J. Véles-Medina, Z. J. Hernández-Estrada and N. Ponce-García, "Viscoelastic properties of tablets from Osborne solubility fraction, pentosans, flour and bread using relaxation tests", Journal of Cereal Science, vol, 69, 2016, pp. 207-212.

[14] N.P. Kozmina, "Biochemistry of bakery", Moskva Food industry, 1978, $277 \mathrm{p}$

[15] V.L. Kretovich and V.L. Yarovenko, "Enzyme preparations in the food industry", Food Industry, 1975, 535 p.

[16] K. Matsushita, I. Shinya. "Interactions between gliadin adsorbed to the air-water interface and pectin added to the aqueous phase", Journal of Cereal Science,vol. 79, 2018, pp. 201-203. 